\title{
First Resolved Dust Continuum Measurements of Individual Giant Molecular Clouds in
} the Andromeda Galaxy

\author{
Jan Forbrich ${ }^{1,2}$ (1), Charles J. Lada ${ }^{2}$, Sébastien Viaene ${ }^{1,3}$, and Glen Petitpas ${ }^{2}$ \\ ${ }^{1}$ Centre for Astrophysics Research, University of Hertfordshire, College Lane, Hatfield, AL10 9AB, UK \\ ${ }^{2}$ Center for Astrophysics|Harvard \& Smithsonian, 60 Garden Street, MS 72, Cambridge, MA 02138, USA \\ Sterrenkundig Observatorium, Universiteit Gent, Krijgslaan 281, B-9000, Gent, Belgium \\ Received 2019 November 2; revised 2019 December 21; accepted 2020 January 6; published 2020 February 11
}

\begin{abstract}
In our local Galactic neighborhood, molecular clouds are best studied using a combination of dust measurements, to determine robust masses, sizes, and internal structures of the clouds, and molecular-line observations to determine cloud kinematics and chemistry. We present here the first results of a program designed to extend such studies to nearby galaxies beyond the Magellanic Clouds. Utilizing the wideband upgrade of the Submillimeter Array (SMA) at $230 \mathrm{GHz}$, we have obtained the first continuum detections of the thermal dust emission on subGMC scales $(\sim 15 \mathrm{pc})$ within the Andromeda galaxy (M31). These include the first resolved continuum detections of dust emission from individual giant molecular clouds (GMCs) beyond the Magellanic Clouds. Utilizing a powerful capability of the SMA, we simultaneously recorded $\mathrm{CO}(2-1)$ emission with identical $(u, v)$ coverage, astrometry, and calibration, enabling the first measurements of the $\mathrm{CO}$ conversion factor, $\alpha_{\mathrm{CO}}(2-1)$, toward individual GMCs across an external galaxy. Our direct measurement yields an average CO-to-dust mass conversion factor of $\alpha_{\mathrm{CO}-\text { dust }}^{\prime}=0.042 \pm 0.018 M_{\odot}\left(\mathrm{K} \mathrm{km} \mathrm{s}^{-1} \mathrm{pc}^{2}\right)^{-1}$ for the $J=2-1$ transition. This value does not appear to vary with galactocentric radius. Assuming a constant gas-to-dust ratio of 136, the resulting $\alpha_{\mathrm{CO}}=5.7 \pm 2.4 M_{\odot}\left(\mathrm{K} \mathrm{km} \mathrm{s}^{-1} \mathrm{pc}^{2}\right)^{-1}$ for the $2-1$ transition is in excellent agreement with that of GMCs in the Milky Way, given the uncertainties. Finally, using the same analysis techniques, we compare our results with observations of the local Orion molecular clouds, placed at the distance of M31 and simulated to appear as they would if observed by the SMA.
\end{abstract}

Unified Astronomy Thesaurus concepts: Andromeda Galaxy (39); Giant molecular clouds (653); Dust continuum emission (412); CO line emission (262)

\section{Introduction}

Star formation plays a critical role in both galaxy and cosmic evolution. Indeed, it is the rate of star formation that is the primary metric for studies of cosmic evolution (e.g., Madau \& Dickinson 2014). Within galaxies star formation occurs in giant molecular clouds (GMCs) on scales of several tens of parsecs. The physical conditions within these clouds set the star formation rate (SFR) for both the clouds and the galaxies that contain them. Understanding the physical nature of GMCs is thus key to understanding the physical processes that drive star formation, set the SFR, and thus determine the evolution of galaxies.

GMCs are almost entirely composed of molecular hydrogen and atomic helium gas, mixed with a sprinkling of interstellar dust. These components account for approximately $75 \%, 24 \%$, and $1 \%$ of the total cloud mass, respectively in Milky Way (MW) GMCs. Yet, since their discovery nearly five decades ago, almost everything we know about the nature of GMCs has been derived from observations of rare, trace molecular species, most notably $\mathrm{CO}$, which account for only about $\sim 0.03 \%$ of the total mass of such clouds (e.g., Heyer \& Dame 2015). Measurements of even such fundamental cloud properties as size, mass, and internal structure have been traditionally hampered by uncertainties inherent in such observations, including variations in molecular abundances, opacities, and excitation conditions within and between the clouds.

However, after decades of slow progress in MW molecular cloud research dominated by observations of rare gas tracers such as $\mathrm{CO}$, the field was transformed when observational capabilities were developed that enabled reliable measurements of the dust in GMCs. Near-infrared, wide-field imaging surveys produced exquisite dust extinction maps of nearby clouds, providing unprecedented data on cloud structure and the first robust and consistently reliable masses of local GMCs (e.g., Lada et al. 1994, 2010; Alves et al. 2001; Goodman et al. 2009). The Herschel and Planck missions have produced widearea surveys of dust continuum emission, greatly improving on the sensitivity, resolution, and dynamic range of measurements of dust (and total) column density in Galactic GMCs (e.g., André et al. 2010; Lombardi et al. 2014; Planck Collaboration et al. 2014a; Peretto et al. 2016).

Since molecular hydrogen forms on the surface of dust grains (e.g., Wakelam et al. 2017), dust is expected to be very well mixed with molecular hydrogen gas. Moreover, measurements of the dust can provide more reliable cloud mass and structural information than those of molecular lines, since for GMCs in the MW the dust accounts for $\sim 1 \%$ of total cloud masses ( $\sim 30 \times$ that of molecular lines), and measurements of it are not hampered by considerations of excitation, chemistry (e.g., depletion), and opacity that severely hinder molecularline observations. For example, optically thin dust continuum emission can be well described by a modified blackbody (Planck) function that is given by $S_{\nu}=B_{\nu}(T) \tau_{\nu} \Omega_{\nu}$ where $\Omega_{\nu}$ is the beam solid angle, $\tau_{\nu}$ the optical depth, and $B_{\nu}(T)$ the usual Planck formula. Determination of a dust column density therefore depends on only two parameters: the dust temperature $T_{d}$ and the dust opacity $\kappa_{\nu}$. The cloud mass directly follows from knowledge of the distance to the cloud and an assumed gas-to-dust ratio. 
Over the past decade observations of dust absorption and emission in the MW have greatly improved our understanding of GMCs by providing fundamental new insights into their physical nature and relation to star formation. In particular, such observations have (1) provided new clarity in revealing the underlying filamentary nature of GMCs (e.g., André et al. 2010, 2014; Arzoumanian et al. 2011), (2) shown that molecular clouds are characterized by stratified internal structure (e.g., Lada et al. 1999; Alves et al. 2001), (3) demonstrated that cloud probability distribution functions are very well described by power-law functions (Lombardi et al. 2015) whose slopes are correlated with the internal level of star formation activity (Kainulainen et al. 2009; Lada et al. 2009), (4) described a new, improved, star formation law relating the SFR to the amount of dense gas in a cloud (Heiderman et al. 2010; Lada et al. 2010; Evans et al. 2014), (5) dramatically confirmed the mass-size relation of Larson (1981) for clouds, revealing how exquisitely tight this relation is for local GMCs (Lombardi et al. 2010), (6) shown as a result that local GMCs cannot be described by a Kennicutt-Schmidt relation of the type that describes star formation on the global scales of galaxies (Lada et al. 2013), and (7) produced the first detailed measurements of the cloud core mass functions with shapes similar to the stellar initial mass function (e.g., Alves et al. 2007; Könyves et al. 2010).

Molecular gas has been observed in external galaxies for almost as long as it has been studied in the MW. However, due to the enormous distances involved, these studies have been both dominated by observations of $\mathrm{CO}$ (the brightest emitting molecule) and largely restricted to spatial scales (1-10 kpc) much larger that those of individual GMCs. With millimeter/ submillimeter-wave interferometers, such as the Atacama Large Millimeter/submillimeter Array (ALMA), the Plateau de Bure Interferometer, and the Submillimeter Array (SMA), resolved measurements of GMCs are possible in the closest galaxies, and CO studies of GMC populations in these galaxies now approach being placed on a footing similar to that of MW studies (e.g., M51, Colombo et al. 2014; NGC 300, Faesi et al. 2018).

Similar to the situation in the MW, measurements of resolved dust emission from extragalactic GMCs would result in significant advances in our understanding of the nature of these objects. Dust continuum observations on the scales of 100-200 pc in the nearest spiral galaxies, such as M31 and NGC 300, have been enabled by the Herschel and Planck space missions (e.g., Fritz et al. 2012; Riener et al. 2018), but resolved dust continuum observations of GMCs on scales of $\sim 10$ pc have remained elusive until now. Such spatial resolution in the millimeter wavelength range can be achieved using existing interferometers, but so far it has been impossible to reach the required combination of continuum sensitivity, angular resolution, and spatial filtering to detect and resolve dust emission from individual GMCs beyond the Magellanic Clouds.

The recent upgrade of the continuum receivers on the SMA is now providing the first opportunity to obtain resolved measurements of dust emission from individual GMCs in another galaxy: the Andromeda galaxy, M31. At a distance of $780 \mathrm{kpc}, \mathrm{M} 31$ is the nearest large disk galaxy to the Sun. With a bandwidth of currently $32 \mathrm{GHz}$ at an observing frequency of $230 \mathrm{GHz}$ (Grimes et al. 2016), the SMA has the required continuum sensitivity to detect dust emission from GMCs in
M31. Moreover, its $(u, v)$ coverage is such that these GMCs can be perfectly matched to a synthesized beam size of $\sim 10 \mathrm{pc}$ and a maximum angular scale of $\sim 100 \mathrm{pc}$. These capabilities put us in a unique position to detect and resolve, for the first time, the dust emission in individual GMCs from an external galaxy beyond the Magellanic Clouds. Coincidentally, it is in M31 that the first resolved CO measurements of extragalactic GMCs were originally obtained (Vogel et al. 1987; Lada et al. 1988).

Moreover, the $\mathrm{CO}(2-1)$ emission line can be extracted from the wide continuum band both to remove $\mathrm{CO}$ contamination of the expected weak continuum signal from the dust and simultaneously to produce a discrete observation of the $\mathrm{CO}$ gas emission with identical $(u, v)$ coverage, spatial scales, and calibration to the dust observations along with relatively high spectral (velocity) resolution. While it would be possible to select the observing band such that continuum sensitivity is maximized by excluding $\mathrm{CO}$, recording both has several advantages, in spite of the challenges due to fact that the dust continuum emission is very faint in comparison. Most importantly, due to the higher relative sensitivity, the CO data can be used to delineate the clouds and determine their structure, which in turn helps us to identify any contaminant continuum emission that is not related to clouds.

Given the fortuitous match of the spatial resolution, spatial filtering, and sensitivity of the SMA to relevant absolute scales at the distance of M31, and the latitude of the SMA coupled with M31's northern decl., it would be hard to replicate these capabilities at any other existing observatory. In particular, since ALMA cannot observe M31, the SMA observations we report here open a new and unique window into our most massive, neighboring galaxy.

The Andromeda galaxy is comparable in overall mass, size, and metallicity to the MW but is curiously different in a number of interesting ways. Unlike the MW, M31 has weak spiral structure and its star formation is largely confined to a well-defined ring of molecular gas and dust with a galactocentric radius of $\sim 10 \mathrm{kpc}$, the center of which is offset from the galaxy's center (e.g., Dame et al. 1993; Gordon et al. 2006). This ring is suspected to be the product of a head-on collision between M31 and a smaller galaxy (M32) that took place 210 million years ago (Block et al. 2006). The global SFR is estimated to be between 0.25 and $0.7 M_{\odot} \mathrm{yr}^{-1}$ for M31, a factor of $\sim 2-6$ lower than that estimated for the MW (i.e., 1.65 $M_{\odot} \mathrm{yr}^{-1}$; Licquia \& Newman 2015). This firmly places M31 below the so-called main sequence of star-forming galaxies (e.g., Peng et al. 2010). The total molecular mass of M31 is $\sim 3 \times 10^{8} M_{\odot}$ (Dame et al. 1993; Nieten et al. 2006), which is less than the total molecular mass of the MW (i.e., $10^{9} M_{\odot}$; Heyer \& Dame 2015) by about a factor of three. The most comprehensive studies of M31's star-forming clouds in molecular gas (Nieten et al. 2006) and dust (Kirk et al. 2015) probe scales of $\sim 90 \mathrm{pc}$, sufficient to resolve GMC complexes but not individual GMCs. Resolved measurements of both gas and dust emission from individual GMCs in M31 should lead to new and more detailed tests of our fundamental understanding of both the nature of GMCs and the laws of star formation in galactic disks.

In the following, we describe observations obtained with the SMA, from what is now an ongoing large-scale science program to survey the dust continuum emission of a significant population of GMCs in M31. We describe the first sub-GMC- 
scale detections of dust continuum emission from individual GMCs in M31. We also report the first spatially resolved measurements of dust continuum emission from individual GMCs in an external galaxy beyond the Magellanic Clouds. In this first analysis and paper, we focus on GMC-scale measurements of the $\mathrm{CO}$ conversion factor, $\alpha_{\mathrm{CO}}$, across the galaxy by linking the continuum and $\mathrm{CO}$ measurements. To put these data in context, we describe simulated observations of what the nearby Orion GMCs would look like if we placed them at the distance of M31 and observed them with the upgraded SMA.

\section{Source Selection, Observations, Calibration, and Data Reduction}

Our source selection was based on the results of the Herschel HELGA dust continuum survey. In particular, we selected our target list from the catalog of giant molecular associations (GMAs) in M31 (Kirk et al. 2015). We initially selected 100 candidate regions based on the observed Herschel fluxes and a range of galactocentric radii $(\sim 6-16 \mathrm{kpc})$ of the GMAs to ensure that we sampled potentially strong sources over a representative range of environmental conditions. Over the course of the early survey we found that the likelihood of detecting dust continuum emission on $\sim 15 \mathrm{pc}$ scales was not strongly correlated with the strength of the Herschelemission obtained on $\sim 100 \mathrm{pc}$ scales, nor was it particularly sensitive to galactocentric position.

The observations reported here were carried out with the SMA in its subcompact configuration during two campaigns from UT 2018 August 16 to UT 2018 September 8, then again during the period from UT 2019 September 9 to UT 2019 October 16. Since we expected the CO emission to be strong, we optimized our tuning to minimize the amount of the band contaminated by $\mathrm{CO}$. Observations were performed using RxA and $\mathrm{RxB}$ receivers tuned to local oscillator frequencies of $225.55 \mathrm{GHz}$ and $233.55 \mathrm{GHz}$ respectively, which placed ${ }^{12} \mathrm{CO}$ $J=2-1$ in spectral window $\mathrm{S} 1$ of the RxA upper side band (USB) and ${ }^{13} \mathrm{CO}$ and $\mathrm{C}^{18} \mathrm{O} J=2-1$ in spectral window $\mathrm{S} 1$ of the RxA lower side band (LSB), leaving $\mathrm{RxB}$ free from CO. This configuration provides $32 \mathrm{GHz}$ of continuous bandwidth ranging from 213.55 to $245.55 \mathrm{GHz}$ with a spectral resolution of $140.0 \mathrm{kHz}$ per channel, with the CO contamination contained within only S1 of RxA. For quick looks at the data, RxA S1 was flagged and completely removed from the band to derive $28 \mathrm{GHz}$ of $\mathrm{CO}$-free continuum measurements, but the final images presented here were created using all $32 \mathrm{GHz}$ of the correlator with just the individual ${ }^{12} \mathrm{CO}$ and ${ }^{13} \mathrm{CO}$-contaminated channels of RxA S1 flagged and removed.

Simple experiments comparing in-band fluxes before and after removal of $\mathrm{CO}$ emission show that $\mathrm{CO}$ emission typically accounts for $10 \%-50 \%$ of the total observed flux in the full continuum band, emphasizing the critical importance of removing $\mathrm{CO}$ emission in order to obtain reliable measurements of broadband (dust) continuum emission.

For all observations the nearby quasars $0013+408$ and 0136 +478 were used as the primary phase and amplitude gain calibrators, with absolute flux calibration performed primarily by comparison to Neptune at the start of the observation, and occasionally cross-checked using Uranus at the end of the observation should the early data be of poorer quality. Passband calibration was derived using 3C 454.3. The raw data were smoothed to $1.5 \mathrm{~km} \mathrm{~s}^{-1}$ and calibration was performed using the MIR IDL package for the SMA, with subsequent analysis performed in MIRIAD.

To smooth out any variations in sensitivity caused by weather, generally three targets were observed during a single track (with typically $6.3 \mathrm{hr}$ shared on the targets), with that observation being repeated three times. Using this technique we were able to achieve a relatively uniform rms across all sources of $0.25 \mathrm{mJy}$ or better. For the observing period in 2018, the SMA used all eight antennas in subcompact configuration, resulting in an average synthesized beam of 4 ". $5 \times 3$ "! 8 (or $\sim 15 \mathrm{pc}$ ), whereas during the 2019 campaign one of the two "outer ring" antennas was in the hangar for repairs, resulting in a synthesized beam more of the order of $8^{\prime \prime} \times 5^{\prime \prime}$ (or 30 $\mathrm{pc} \times 19 \mathrm{pc})$. The ${ }^{12} \mathrm{CO}(2-1)$ and ${ }^{13} \mathrm{CO}(2-1)$ transitions were excised from the wideband data and imaged separately. For this study we produced integrated intensity, moment 0 , maps of the excised ${ }^{12} \mathrm{CO}(2-1)$ data, and we defer a full discussion of the $\mathrm{CO}$ data to a follow-up paper.

\section{Data Analysis and Results}

In this paper our analysis will focus on comparing the dust continuum detections with corresponding $\mathrm{CO}$ emission to measure the $\mathrm{CO}$ conversion factor, $\alpha_{\mathrm{CO}}$, for the cloud sample thus far observed in our program.

For this purpose, we have analyzed images of the continuum data after removing the small CO-emitting portions of the continuum band. We look for continuum emission above a significance limit of $3 \sigma$, where the sensitivity achieved in the individual observations varies slightly (see Section 2). In our initial sample, all but one of our targets (K161) show significant dust continuum emission. The ability to separately excise potentially contaminating $\mathrm{CO}(2-1)$ emission from the wideband SMA data allows us to use both continuum and separate CO images to identify and characterize the GMCs. Since the relative sensitivity is far greater in $\mathrm{CO}$ than in the dust continuum, the $\mathrm{CO}$ moment 0 maps represent the best resolved information on the location, size, and shape of the GMCs in the sample, resolving the Herschel GMA candidates into one or several clouds. The $\mathrm{CO}$ and continuum maps also have identical astrometry, and the CO maps thus also generally set the outer bounds for continuum detections within the confines of a GMC. As an example, the resulting continuum images for nine targets with resolved continuum detections are shown in Figure 1 along with the corresponding contours of $\mathrm{CO}$ emission. Given the unusual fractional bandwidth of these observations, we consider a source to be resolved if the emitting area of significant dust emission is larger than the synthesized beam by a factor of 1.2. In most cases the $\mathrm{CO}$ emission is more extended than the dust emission, as expected given the current disparity in relative sensitivities to cloud material of the two tracers. The coincidence or nearcoincidence of peak continuum emission with peak $\mathrm{CO}$ emission confirms that we are observing emission from dust at these positions in the clouds.

To facilitate a direct comparison of dust and $\mathrm{CO}$ emission, we use the regions with significant continuum emission to define masks for the extraction of the corresponding $\mathrm{CO}$ data. In this way, we extract corresponding continuum and $\mathrm{CO}$ fluxes for all targets. We express the $\mathrm{CO}$ fluxes in units of $I_{\mathrm{CO}}($ i.e., $\mathrm{K} \mathrm{km} \mathrm{s}^{-1}$ ). Many of the sources have several spatially distinct detections of significant dust continuum emission, and once identified we assess these individual detections separately, 

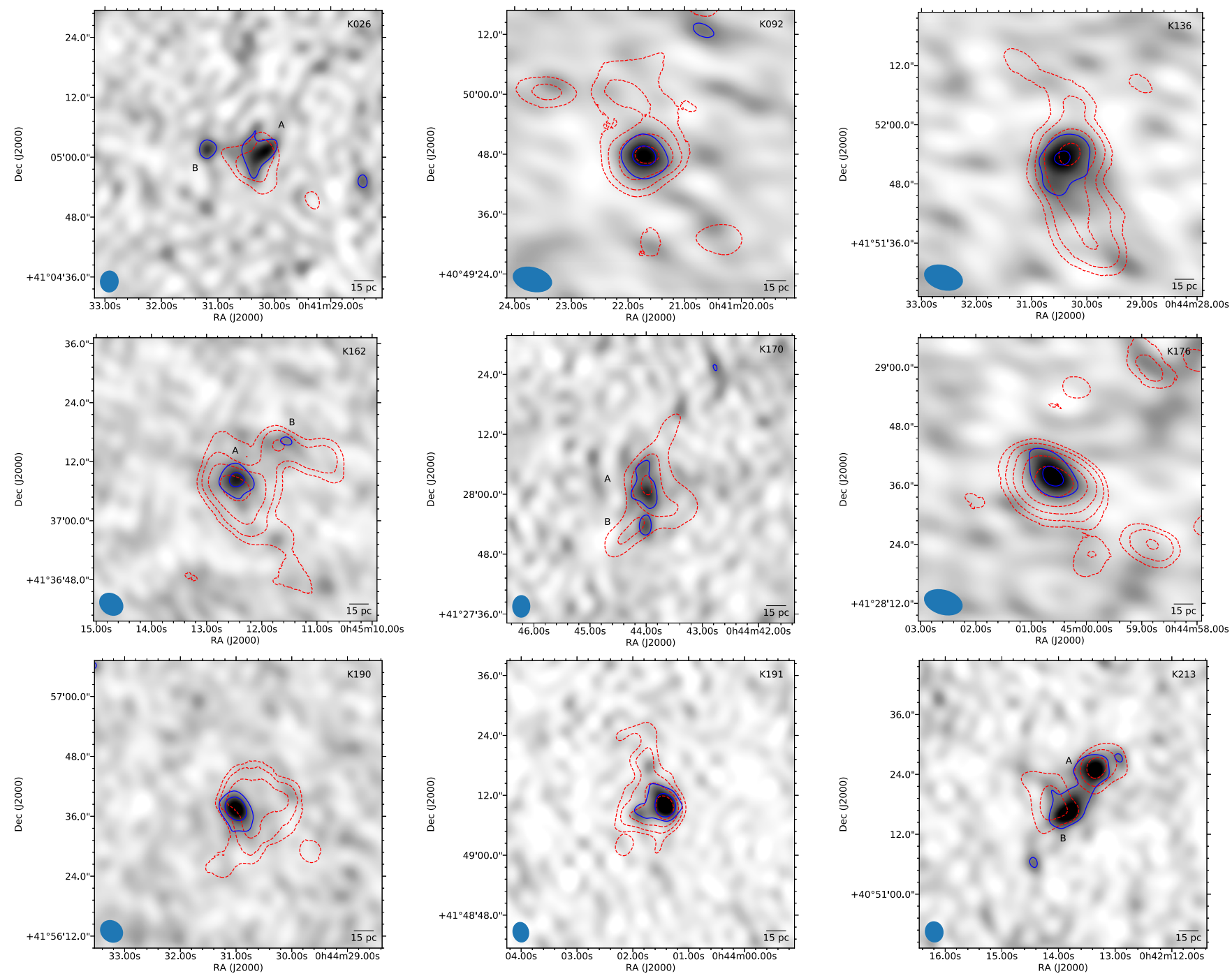

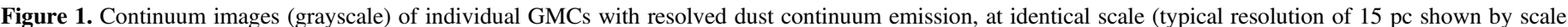

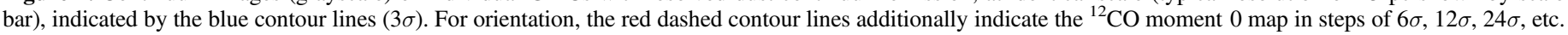
The synthesized beam size is indicated in the lower left corner of each panel.

labeling the resulting individual sources in each target image with letters (A, B, C, etc.). Out of a total of 15 initial targets included here, we thus end up with a total of 23 continuum sources. For each of these, we obtain measurements of the integrated continuum and ${ }^{12} \mathrm{CO}(2-1)$ emission, and these are listed in Table 1. For resolved sources, these two measurements are integrated in the same mask, while otherwise unresolved per-beam measurements at identical positions are compared. The continuum flux density for every cloud can be converted into a dust mass, i.e.,

$$
M_{\mathrm{dust}}=S_{\nu} d^{2} /\left(\kappa_{\nu} B_{\nu}\left(T_{d}\right)\right)
$$

To evaluate Equation (1) we need to first specify the dust opacity, $\kappa_{\nu}$, and this requires a choice of a dust model. We adopt the canonical THEMIS MW dust model (Jones et al. 2017), which was calibrated using the Planck data and other observational constraints by Ysard et al. (2015). At $230 \mathrm{GHz}$, this becomes $\kappa_{\nu}=0.0425 \mathrm{~m}^{2} \mathrm{~kg}^{-1}$. The THEMIS dust model proved to work well in the radiative transfer model of M31 by Viaene et al. (2017), which links dust extinction to dust emission. However, the kind of dust model most suitable for
M31 is still a matter of debate (see, e.g., Whitworth et al. 2019).

Assuming a dust temperature of $T_{d}=20 \mathrm{~K}$ and a distance of $780 \mathrm{kpc}$, the dust masses for the clouds calculated from Equation (1) with the above assumptions range from 181 to $1825 M_{\odot}$. To obtain total cloud masses requires knowledge of the gas-to-dust ratio, $R_{g / d}$, that is, $M_{\text {tot }}=M_{\text {dust }} \times R_{g / d}$. Assuming a gas-to-dust ratio of $\sim 136$ to compare with the MW would result in a range of total cloud masses of about 24,000-240,000 $M_{\odot}$. For a different assumed gas-to-dust ratio the total mass would be $M_{\mathrm{tot}}^{\prime}=M_{\mathrm{tot}} \times\left(R_{g / d} / 136\right)$.

\section{Discussion}

As described above, observations of dust in both absorption and emission provide the best tool for measuring many of the most fundamental properties of GMCs and star formation. However, in many situations, such as in the distant universe and even the more remote regions of the $\mathrm{MW}, \mathrm{CO}$ will remain the best available tracer of GMCs and the molecular interstellar medium for the foreseeable future. To be able to make full use of its capabilities as a tracer of molecular gas it is essential to be 
Table 1

Cloud Sample and Properties

\begin{tabular}{|c|c|c|c|c|c|c|}
\hline (1) & $\begin{array}{c}F_{\text {cont }} \\
(\mathrm{mJy}) \\
(2)\end{array}$ & $\begin{array}{c}\mathrm{rms} \\
(\mathrm{mJy}) \\
(3)\end{array}$ & $\begin{array}{c}I_{\mathrm{CO}} \\
\left(\mathrm{K} \mathrm{km} \mathrm{s}^{-1}\right) \\
(4)\end{array}$ & $\begin{array}{c}M_{\text {dust }} \\
\left(M_{\odot}\right) \\
(5)\end{array}$ & $\left.M_{\odot} \underset{(6)}{\alpha_{\mathrm{CO}-\text { dust }}^{\prime} \mathrm{km} \mathrm{s}^{-1}} \mathrm{pc}^{2}\right)^{-1}$ & $\begin{array}{c}R_{\text {gal }} \\
(\mathrm{kpc}) \\
(7)\end{array}$ \\
\hline K026A & $1.86 \pm 0.31$ & 0.23 & $20.4 \pm 1.1$ & $529 \pm 89$ & $0.035 \pm 0.008$ & 5.8 \\
\hline K026B & $1.17 \pm 0.23$ & 0.23 & $6.0 \pm 0.8$ & $333 \pm 65$ & $0.150 \pm 0.040$ & 5.8 \\
\hline K048 & $0.86 \pm 0.27$ & 0.27 & $18.0 \pm 1.1$ & $244 \pm 77$ & $0.040 \pm 0.013$ & 5.7 \\
\hline K092 & $1.43 \pm 0.25$ & 0.21 & $11.5 \pm 0.9$ & $406 \pm 71$ & $0.027 \pm 0.007$ & 8.0 \\
\hline K093A & $1.13 \pm 0.20$ & 0.20 & $11.0 \pm 0.7$ & $322 \pm 58$ & $0.084 \pm 0.021$ & 8.1 \\
\hline К093В & $0.64 \pm 0.20$ & 0.20 & $13.5 \pm 0.7$ & $182 \pm 58$ & $0.039 \pm 0.012$ & 8.1 \\
\hline K134 & $1.27 \pm 0.31$ & 0.31 & $7.3 \pm 1.8$ & $362 \pm 88$ & $0.051 \pm 0.021$ & 10.5 \\
\hline K136 & $2.17 \pm 0.36$ & 0.25 & $10.5 \pm 1.6$ & $617 \pm 101$ & $0.031 \pm 0.010$ & 11.3 \\
\hline K157 & $1.70 \pm 0.31$ & 0.31 & $12.6 \pm 1.3$ & $483 \pm 89$ & $0.039 \pm 0.009$ & 11.9 \\
\hline K160A & $0.99 \pm 0.22$ & 0.22 & $5.5 \pm 0.9$ & $282 \pm 62$ & $0.053 \pm 0.015$ & 12.3 \\
\hline K160B & $0.70 \pm 0.22$ & 0.22 & $7.5 \pm 0.9$ & $200 \pm 62$ & $0.027 \pm 0.009$ & 12.3 \\
\hline K162A & $1.20 \pm 0.22$ & 0.19 & $27.7 \pm 0.8$ & $342 \pm 62$ & $0.017 \pm 0.004$ & 11.8 \\
\hline K162B & $0.64 \pm 0.19$ & 0.19 & $14.9 \pm 0.7$ & $181 \pm 53$ & $0.024 \pm 0.007$ & 11.8 \\
\hline K170A & $1.47 \pm 0.29$ & 0.23 & $27.0 \pm 1.5$ & $417 \pm 84$ & $0.024 \pm 0.006$ & 11.8 \\
\hline K170B & $1.00 \pm 0.23$ & 0.23 & $17.6 \pm 1.1$ & $285 \pm 65$ & $0.043 \pm 0.011$ & 11.8 \\
\hline K176 & $1.71 \pm 0.29$ & 0.24 & $11.3 \pm 0.6$ & $486 \pm 84$ & $0.030 \pm 0.007$ & 13.8 \\
\hline K190 & $1.63 \pm 0.22$ & 0.24 & $4.6 \pm 0.2$ & $463 \pm 63$ & $0.122 \pm 0.020$ & 13.9 \\
\hline K191 & $6.41 \pm 0.71$ & 0.17 & $34.5 \pm 0.7$ & $1826 \pm 202$ & $0.053 \pm 0.007$ & 12.1 \\
\hline K213A & $3.91 \pm 0.52$ & 0.31 & $22.2 \pm 0.7$ & $1115 \pm 147$ & $0.049 \pm 0.008$ & 12.0 \\
\hline K213B & $3.37 \pm 0.46$ & 0.31 & $14.2 \pm 0.6$ & $959 \pm 131$ & $0.085 \pm 0.015$ & 12.0 \\
\hline $\mathrm{K} 213 \mathrm{C}$ & $1.03 \pm 0.31$ & 0.31 & $16.9 \pm 0.4$ & $294 \pm 89$ & $0.048 \pm 0.015$ & 12.0 \\
\hline K291A & $1.09 \pm 0.22$ & 0.22 & $5.1 \pm 0.2$ & $312 \pm 62$ & $0.162 \pm 0.036$ & 16.0 \\
\hline K291B & $0.69 \pm 0.22$ & 0.22 & $1.3 \pm 0.2$ & $196 \pm 62$ & $0.410 \pm 0.132$ & 16.0 \\
\hline
\end{tabular}

able to calibrate its effectiveness by direct comparison with observations of dust across as wide a range of conditions as possible.

\subsection{Measuring $\alpha_{\mathrm{CO}}$ in M31 GMCs}

Observations of $\mathrm{CO}$ are most frequently used to derive molecular gas and cloud masses. In particular, it is assumed that the luminosity of $\mathrm{CO}$ from a region of space is directly proportional to the mass of molecular gas in that region. This is often expressed as $M_{\mathrm{tot}}=\alpha_{\mathrm{CO}} \times L_{\mathrm{CO}}$. Here $M_{\mathrm{tot}}$ is the total molecular mass, including a $36 \%$ correction for the presence of heavier elements (i.e., helium, etc.) and has units of solar masses $\left(M_{\odot}\right), \alpha_{\mathrm{CO}}$ is the $\mathrm{CO}$ conversion factor and has units of $M_{\odot}\left(\mathrm{K} \mathrm{km} \mathrm{s}^{-1} \mathrm{pc}^{2}\right)^{-1}$, and $L_{\mathrm{CO}}$ is the $\mathrm{CO}$ luminosity expressed in units of $\mathrm{K} \mathrm{km} \mathrm{s}^{-1} \mathrm{pc}^{2}$. The value of $\alpha_{\mathrm{CO}}$ for the $1-0$ transition of $\mathrm{CO}$ has been derived both theoretically (using virial arguments) and empirically (often from direct comparison with dust), and it is estimated to have a value of $4.3 M_{\odot}(\mathrm{K}$ $\left.\mathrm{km} \mathrm{s}^{-1} \mathrm{pc}^{2}\right)^{-1}$ in the MW and throughout the local universe (Bolatto et al. 2013). This corresponds to a value of $6.1 M_{\odot}(\mathrm{K}$ $\left.\mathrm{km} \mathrm{s}^{-1} \mathrm{pc}^{2}\right)^{-1}$ for the $J=2-1$ transition of $\mathrm{CO}$, assuming a typical intensity ratio of $I_{\mathrm{CO}}(2-1) / I_{\mathrm{CO}}(1-0)$ of 0.7 . Variations in $\alpha_{\mathrm{CO}}$ have been reported in the nuclear regions of the $\mathrm{MW}$ and other galaxies and are expected in regions of varying metallicity (Bolatto et al. 2013 and references therein).

Our observations of dust and $\mathrm{CO}$ in $\mathrm{M} 31$ provide an opportunity to measure $\alpha_{\mathrm{CO}}$ on sub-GMC scales across the Andromeda galaxy. Measuring the value of $\alpha_{\mathrm{CO}}$ is straightforward from our data, given the identical spatial sensitivity, astrometry, and calibration of the dust continuum and $\mathrm{CO}$ data.

Our most direct measurement is that of a conversion factor from $\mathrm{CO}$ luminosity to dust mass, $\alpha_{\mathrm{CO}-\text { dust }}^{\prime}$, since it does not depend on the choice of a gas-to-dust ratio. We first obtain $\mathrm{CO}$ measurements that correspond to the mask where significant dust continuum emission has been detected, with the initial hypothesis that all of these objects may be GMCs. By calculating the CO luminosity in this same mask, we can then directly measure the $\alpha_{\mathrm{CO} \text {-dust }}^{\prime}$ parameter. In the case of multiple dust continuum peaks per target, we consider each of these separately. The results are shown in Table 1 and displayed in Figure 2. In spite of a considerable range in galactocentric radius already in this preliminary sample, we find that the clouds can be described by a single value of $\alpha_{\mathrm{CO} \text {-dust }}^{\prime}$. Excluding the four most extreme outliers, which are likely contaminants (see below), we find a mean value of $\alpha_{\mathrm{CO}-\text { dust }}^{\prime}=0.042 \pm 0.018 M_{\odot}\left(\mathrm{K} \mathrm{km} \mathrm{s}^{-1} \mathrm{pc}^{2}\right)^{-1}$. Assuming a gas-to-dust ratio of $\sim 136$ to compare with MW clouds, this value corresponds to $\alpha_{\mathrm{CO}(\mathrm{M} 31)}=5.7 \pm 2.4 M_{\odot}\left(\mathrm{K} \mathrm{km} \mathrm{s}^{-1}\right.$ $\left.\mathrm{pc}^{2}\right)^{-1}$. Within the uncertainties our result is in good agreement with the Galactic value of $\alpha_{\mathrm{CO}(\mathrm{Gal})}=6.1 M_{\odot}\left(\mathrm{K} \mathrm{km} \mathrm{s}^{-1} \mathrm{pc}^{2}\right)^{-1}$ for the 2-1 transition of CO (Bolatto et al. 2013). Our result is also compatible with the study of Leroy et al. (2011), whose estimates of $\alpha_{\mathrm{CO}}(1-0)$ on kiloparsec scales in M31 were also reported to be consistent with the MW value.

These considerations depend on the assumptions spelled out above, most notably the assumed dust temperature (see Equation (1)). While all our target GMAs have dust temperatures determined by the HELGA survey, as listed in Kirk et al. (2015), these estimates are limited by the angular resolution of the Herschel SPIRE $350 \mu \mathrm{m}$ band at $\sim 24^{\prime \prime}$ or $\sim 90 \mathrm{pc}$, smoothing out a lot of the temperature structure present at resolutions of $\sim 15 \mathrm{pc}$. We have thus decided to assume a common typical dust temperature of $T_{d}=20 \mathrm{~K}$. However, the impact of different temperatures on the determination of $\alpha_{\mathrm{CO}}$ is limited. This is shown in Figure 2, where we additionally plot the $\alpha_{\mathrm{CO}-\text { dust }}^{\prime}$ values for assumed temperatures of $T_{d}=15 \mathrm{~K}$ and $T_{d}=25 \mathrm{~K}$. 


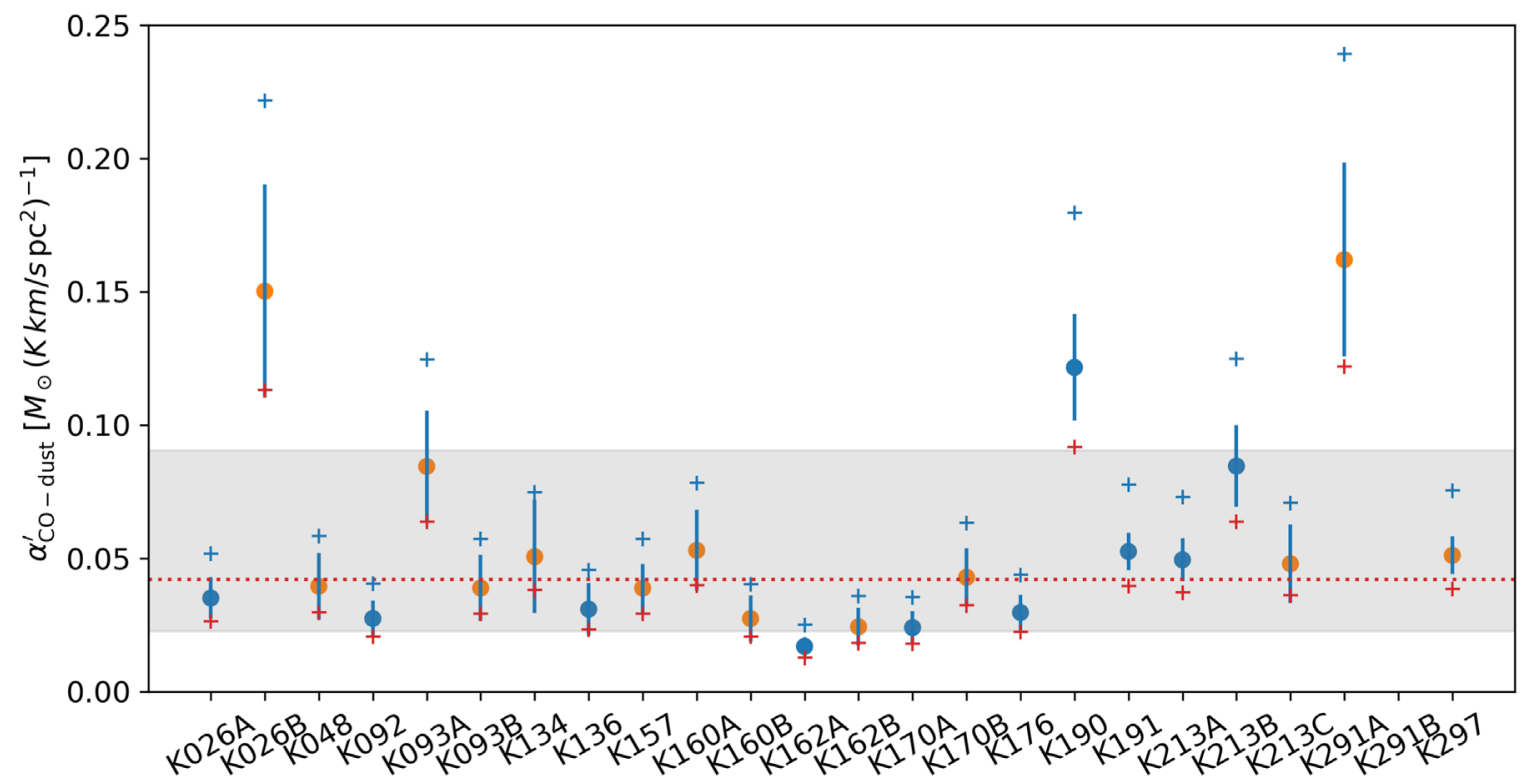

Figure 2. Direct $\alpha_{\mathrm{CO}-\text { dust }}^{\prime}$ measurements per cloud, converting $\mathrm{CO}$ luminosity to dust mass. Blue dots indicate resolved sources, and orange dots indicate unresolved sources, both for $T_{d}=20 \mathrm{~K}$. Red and blue crosses additionally show $\alpha_{\mathrm{CO}}$ for $T_{d}=25 \mathrm{~K}$ and $T_{d}=15 \mathrm{~K}$, respectively. The dotted line indicates the (clipped, see text) sample mean of $\alpha_{\mathrm{CO} \text {-dust }}^{\prime}=0.042 M_{\odot}\left(\mathrm{K} \mathrm{km} \mathrm{s}^{-1} \mathrm{pc}^{-2}\right)^{-1}$. For comparison, we divide the nominal Galactic value for the $\mathrm{CO}(2-1)$ transition (see text) by a gas-to-dust ratio of 136 to find a near-identical value of $\alpha_{\mathrm{CO}-\text { dust(Gal })}^{\prime}=0.045 M_{\odot}\left(\mathrm{K} \mathrm{km} \mathrm{s}^{-1} \mathrm{pc}^{-2}\right)^{-1}$, and we indicate the corresponding range with an uncertainty of \pm 0.3 dex as a gray shaded area. Note that K291B is off-scale (see Table 1).

Nonetheless, given our assumptions, our measurements are compatible with a constant $\alpha_{\mathrm{CO}}^{\prime}$ value for the M31 GMC population we have so far sampled. This value is in excellent agreement with that of MW GMCs (i.e., 6.1/136 = 0.045) and is quite interesting given the considerable range in galactocentric radii $(6-12 \mathrm{kpc})$ and presumably environments spanned by our target GMCs in M31. Similarly, our derived estimate for $\alpha_{\mathrm{CO}}$ also is compatible with a constant value, provided the gasto-dust ratio is constant in the regions we have observed.

Finally, we note that there are also a few outliers with seemingly very high $\alpha_{\mathrm{CO}}^{\prime}$. These are sources where unresolved continuum emission is detected in areas of only very faint or no $\mathrm{CO}$ emission, in spite of the high sensitivity of the $\mathrm{CO}$ observations. As we discuss below, these objects are real, and likely contaminants of differing physical nature from the sources of dust emission within the GMCs.

\subsection{Nature of the Contaminating Sources of Continuum Emission}

The common astrometry of both data sets enables us to spatially match continuum peaks to structure in the CO maps with a high degree of confidence that would be difficult to duplicate in experiments where the two data sets were obtained at different epochs or with different telescopes. This is a great advantage because it not only allows accurate association of dust and $\mathrm{CO}$ emission, but also enables confident identification of any continuum sources not spatially related to the GMCs traced by CO. For example, in a few of our images we find continuum peaks that are offset from the locations of the COemitting gas. The much higher relative sensitivity of the $\mathrm{CO}$ data to cloud material suggests that such sources are not associated with GMCs in M31 and are real contaminants of a different physical nature or class than the dust sources we detect within the GMCs. Without the angular resolution and common astrometry provided by the SMA, such continuum emission could be falsely associated with the $\mathrm{CO}$ emission.
Potential sources of "contamination" include the direct detection of free-free emission from luminous H II regions, even at $230 \mathrm{GHz}$, and the detection of background galaxies. While the latter can only be corroborated if suitable additional data are available, we can at least approximately assess the incidence of $\mathrm{H}$ II regions whose free-free emission is bright enough to be detected at $230 \mathrm{GHz}$. As a first estimate, we can scale the centimeter-wavelength free-free flux density of the Orion Nebula to the distance of M31, applying a spectral index of -0.1 for optically thin free-free emission to extrapolate to $230 \mathrm{GHz}$. The total flux density of this relatively puny H II region of $\sim 400 \mathrm{Jy}$ (e.g., Felli et al. 1993) corresponds to an unresolved source of $0.09 \mathrm{mJy}$ at the distance of M31, and it is thus not detectable in our observations. However, a source with just seven times the flux density of the Orion Nebula would be marginally detectable at $3 \sigma$, assuming an rms sensitivity of $0.20 \mathrm{mJy}$. Next, we now place the Orion Nebula into the Galactic context. While no unbiased catalog of H II regions exists for either the MW or M31, we can use the catalog obtained by Murray \& Rahman (2010) in an all-sky survey for free-free emission from $\mathrm{H}$ II regions based on data obtained by the Wilkinson Microwave Anisotropy Probe. They list the freefree luminosities of 183 Galactic H II regions. Even though necessarily incomplete, this list contains 174 regions that are more luminous than the Orion Nebula, and 149 regions that are at least seven times and up to three orders of magnitude more luminous. While the full Galactic context remains unclear, the detection, in our observations, of free-free emission from H II regions at $230 \mathrm{GHz}$ and in M31 is thus evidently possible.

The four outliers in our $\alpha_{\mathrm{CO}}$ results, namely K026B, K190, and $\mathrm{K} 291 \mathrm{~A} / \mathrm{B}$, are candidates for non-dust continuum emission. Out of these, K026B has very little CO emission, and it coincides with a faint X-ray source (Wang et al. 2016) and a faint red Spitzer source (Khan 2017). It thus seems plausible that $\mathrm{K} 026 \mathrm{~B}$ is in fact a background galaxy. The other three sources will require further analysis to establish their nature. 


\subsection{Context: Orion GMCs at the Distance of M31}

To assess the types of clouds that we are detecting in M31, it is helpful to put the results into the context of the local clouds. How would the Orion molecular clouds appear in our observations if they were located in M31? To answer this question, we use existing dust continuum and CO maps of the Orion clouds. The Planck satellite has provided us with all-sky maps that are ideal for this purpose. For a simulation that is as close as possible to our actual observations, we opt for the map at $217 \mathrm{GHz}$. These are bolometric maps with a much wider effective bandwidth than the SMA, but they are ideal for a general test of feasibility in terms of both signal-to-noise ratio $(\mathrm{S} / \mathrm{N})$ and spatial recovery of the cloud. In a first step, we convert the units of the Planck map from $\mathrm{K}(\mathrm{CMB})$ to $\mathrm{MJy} \mathrm{sr}^{-1}$. Given the wide Planck band, different spectral energy distributions (SEDs) can make a difference in this conversion, either in the form of a correction factor or as a change in the effective frequency. In the case of a typical dust SED, the correction is accomplished by a shift in effective frequency to $229 \mathrm{GHz}$, which is perfect for our purposes. Alternatively, at the same reference frequency, the correction factor is only 0.85 (Planck Collaboration et al. 2014b). The map is then converted into $\mathrm{Jy} \mathrm{beam}^{-1}$ before we convert the pixel scale, astrometry, and beam size of the map to reflect the distance of M31 rather than Orion (414 pc; Menten et al. 2007). A direct comparison in $\mathrm{CO}(2-1)$ is provided by Nishimura et al. (2015), who mapped the Orion clouds in various molecular transitions with a $1.85 \mathrm{~m}$ dish. ${ }^{4}$

For our purposes of simulating observations of Orion-like clouds in M31, both data sets have far better angular resolution than required and are thus ideal. To simulate observations, we first converted the Planck data from $\mathrm{K}(\mathrm{CMB})$ to $\mathrm{Jy} \mathrm{beam}^{-1}$ by applying a conversion factor of $483.690 \mathrm{MJy} \mathrm{sr}^{-1}$ (Planck Collaboration et al. 2014b) at an effective frequency of $229 \mathrm{GHz}$ (see above), and assuming a beam size of $5^{\prime}$ (FWHM). Similarly the CO data were converted from main beam temperatures to flux densities. Both maps were then scaled to the distance of M31 of $780 \mathrm{kpc}$, requiring changes to the flux densities, pixel sizes, and beam sizes. In order to achieve realistic simulations, we additionally changed the source coordinates for Orion to those of M31, such that the simulated $(u, v)$ coverage is comparable to that of our actual M31 observations.

The resulting images were then used as input for the simulated observations in CASA 5.3, using the tasks simobserve and simanalyze. In these simulations, we assume full tracks with the subcompact configuration of the SMA to ensure realistic $(u, v)$ coverage. The simulated continuum images of Orion, observed with the SMA as if it were located in M31 are shown in Figure 3 and compared to the detectable extent of $\mathrm{CO}$ emission from this region. The left panel of Figure 3 shows the input Planck $213 \mathrm{GHz}$ continuum emission and the right panel the simulated continuum observations. The blue contours show the depths that would be reached for integration times corresponding to one, two, and four interferometer tracks, assuming a one-track $3 \sigma$ sensitivity of $0.6 \mathrm{mJy}^{\text {beam }}{ }^{-1}$. With one track we are able to detect the Ori A cloud at the $3 \sigma$ level as an unresolved source. Our simulated one-track detection corresponds to a dust mass of $216 \pm 32 M_{\odot}$, which for $R_{g / d}=136$ (appropriate for Orion) corresponds to $2.93 \times 10^{4}$

\footnotetext{
4 See also http://www.astro.s.osakafu-u.ac.jp/ nishimura/Orion/.
}

$M_{\odot}$, roughly $40 \%$ of the total cloud mass reported by Lada et al. (2010) for the Orion A GMC. This corresponds to the total cloud mass known to be above an extinction of $A_{V} \approx 4.0$ magnitudes.

These simulations suggest that continuum sources at our limit of detection in M31 are comparable in scale to the local GMCs. Moreover, since the dynamic range in dust emission in GMCs is not very high, our simulations suggest the exciting possibility that one would not have to go too much deeper (two or three additional tracks) to be able to detect dust emission with the SMA from a very significant fraction, if not the entirety, of a GMC in M31. The simulations also add support to the notion that the $\mathrm{CO}$ is detected a lot more easily than the continuum, and that one $\mathrm{CO}$ track is sufficient to detect and define the outer boundary of the clouds.

Finally, we obtain $\alpha_{\mathrm{CO}}$ measurements for these simulated observations of the Orion A cloud in M31, using the same procedure that we also applied to our actual observations. For Orion A we find $\alpha_{\mathrm{CO}-\text { dust }}^{\prime}=0.038 \pm 0.006 M_{\odot}\left(\mathrm{K} \mathrm{km} \mathrm{s}^{-1}\right.$ $\left.\mathrm{pc}^{2}\right)^{-1}$, corresponding to $\alpha_{\mathrm{CO}}=5.2 \pm 0.8 M_{\odot}\left(\mathrm{K} \mathrm{km} \mathrm{s}^{-1}\right.$ $\left.\mathrm{pc}^{2}\right)^{-1}$, assuming a gas-to-dust ratio of 136. This is compatible with standard Galactic values and those we find in M31. Comparison of our simulations with our observations reinforces the notion that the basic physical nature of GMCs in M31 is very similar to that of GMCs in the local region of the MW.

\section{Summary and Conclusions}

We have taken advantage of the upgrade to the SMA wideband receiver to obtain the first continuum detections of the thermal dust emission on sub-GMC scales $(\sim 15 \mathrm{pc})$ within individual GMCs in the Andromeda galaxy. These include the first resolved continuum detections of dust emission from individual GMCs beyond the Magellanic Clouds.

We detected 23 discrete continuum sources toward 15 target regions. Of these, 19 objects are shown to be sources of thermal dust emission from within individual GMCs; 10 of these sources are resolved. Derived dust masses range from approximately 200 to $1800 M_{\odot}$. We also simultaneously obtained high-quality in-band measurements of $\mathrm{CO}(2-1)$ emission from each region with the same $(u, v)$ coverage, astrometry, and calibration as the continuum observations. We combined these measurements to make direct determinations of the $\mathrm{CO}$ conversion factor, $\alpha_{\mathrm{CO}}$, in individual GMCs across the disk of M31. Our direct determination of a conversion factor from $\mathrm{CO}$ luminosity to dust mass yields

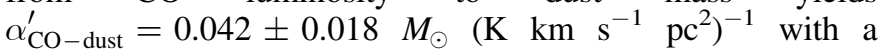
relatively small dispersion. In particular, this value does not appear to be a function of galactocentric radius (at least over a range from 6 to $12 \mathrm{kpc}$ ). Assuming a gas-to-dust ratio of $\sim 136$ for comparison with the MW clouds, we find a corresponding $\alpha_{\mathrm{CO}}=5.7 \pm 2.4 M_{\odot}\left(\mathrm{K} \mathrm{km} \mathrm{s}^{-1} \mathrm{pc}^{2}\right)^{-1}$ for the $2-1$ transition of $\mathrm{CO}$, which, within the uncertainties, is in excellent agreement with the value (6.1) previously found to characterize MW clouds and the local universe. With this gas-to-dust ratio, the dust-emitting regions in these GMCs have total gaseous masses of 24,000-240,000 $M_{\odot}$. While $\alpha_{\mathrm{CO}-\text { dust }}^{\prime}$ does not depend on the gas-to-dust ratio, our results were derived assuming a constant dust temperature of $20 \mathrm{~K}$ for all the clouds. However, we show that the temperatures can be varied in a reasonable range even within an error budget for $\alpha_{\mathrm{CO}}$ of a factor of two. 

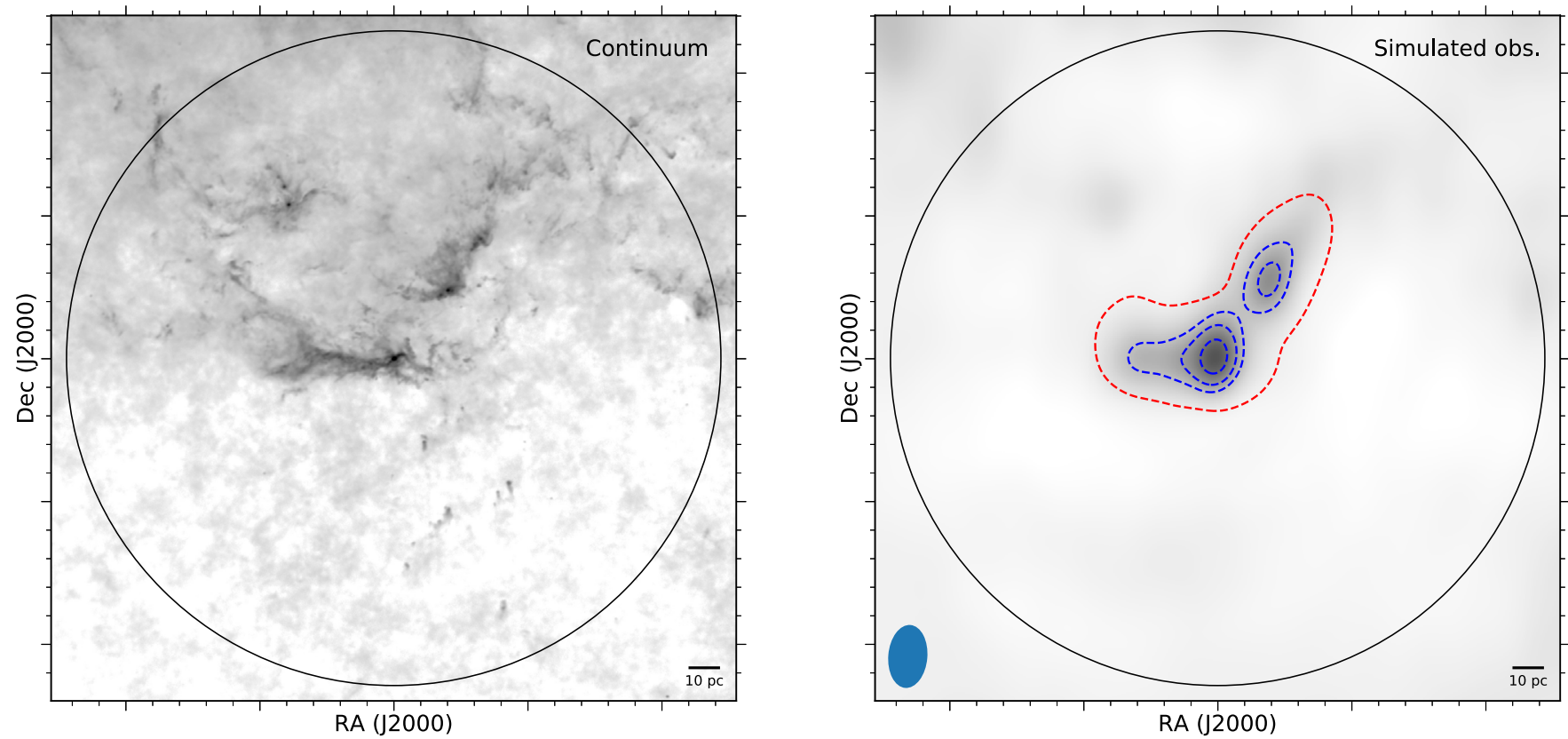

Figure 3. Simulated $230 \mathrm{GHz}$ SMA observations of the Orion molecular clouds, as if they were located in M31, assuming the subcompact configuration, with the half-power beam width of the primary beam indicated (see text). Left: input Planck $217 \mathrm{GHz}$ continuum image. Right: simulated continuum observation, with blue contours indicating a typical $3 \sigma$ per-track sensitivity with $1 \sigma=0.20 \mathrm{mJy}_{\text {beam }}{ }^{-1}$, for one, two, and four tracks with a bandwidth of $32 \mathrm{GHz}$. To visualize these different observing setups in the same image, no artificial noise has been added, such that the simulated image retains the full dynamic range of the model image. The red contour indicates $\mathrm{S} / \mathrm{N}=100$ for a single track of the simulated $\mathrm{CO}(2-1)$ observations.

Additionally, we demonstrate with simulated observations of an Orion-like cloud in M31 that there is meaningful parameter overlap between our sample presented here and the local Galactic GMCs. With these results in hand and a growing sample of clouds being observed with the SMA, it will finally be possible to place our understanding of the nature of the GMC population of an external galaxy, such as M31, on a similar footing to that of the well studied GMCs in the local MW.

We thank Chris Faesi for insightful discussions and assistance with verification of $\mathrm{CO}$ reduction procedures. The Submillimeter Array is a joint project between the Smithsonian Astrophysical Observatory and the Academia Sinica Institute of Astronomy and Astrophysics and is funded by the Smithsonian Institution and the Academia Sinica.

Facility: Submillimeter Array.

Software: MIRIAD (Sault et al. 1995), MIR IDL package (https://www.cfa.harvard.edu/rtdc/SMAdata/process/mir/), CASA (McMullin et al. 2007).

\section{ORCID iDs}

Jan Forbrich (1) https://orcid.org/0000-0001-8694-4966

\section{References}

Alves, J., Lombardi, M., \& Lada, C. J. 2007, A\&A, 462, L17 Alves, J. F., Lada, C. J., \& Lada, E. A. 2001, Natur, 409, 159 André, P., Di Francesco, J., Ward-Thompson, D., et al. 2014, in Protostars and Planets VI, ed. H. Beuther et al. (Tucson, AZ: Univ. Arizona Press), 27 André, P., Men'shchikov, A., Bontemps, S., et al. 2010, A\&A, 518, L102 Arzoumanian, D., André, P., Didelon, P., et al. 2011, A\&A, 529, L6 Block, D. L., Bournaud, F., Combes, F., et al. 2006, Natur, 443, 832 Bolatto, A. D., Wolfire, M., \& Leroy, A. K. 2013, ARA\&A, 51, 207 Colombo, D., Hughes, A., Schinnerer, E., et al. 2014, ApJ, 784, 3 Dame, T. M., Koper, E., Israel, F. P., \& Thaddeus, P. 1993, ApJ, 418, 730 Evans, N. J., II, Heiderman, A., \& Vutisalchavakul, N. 2014, ApJ, 782, 114
Faesi, C. M., Lada, C. J. \& Forbrich, J. 2018, ApJ, 857, 19

Felli, M., Churchwell, E., Wilson, T. L., \& Taylor, G. B. 1993, A\&AS, 98, 137 Fritz, J., Gentile, G., Smith, M. W. L., et al. 2012, A\&A, 546, A34

Goodman, A. A., Pineda, J. E., \& Schnee, S. L. 2009, ApJ, 692, 91

Gordon, K. D., Bailin, J., Engelbracht, C. W., et al. 2006, ApJL, 638, L87

Grimes, P., Blundell, R., Paine, S., Tong, C. Y. E., \& Zeng, L. 2016, Proc. SPIE, 9914, 991424

Heiderman, A., Evans, N. J., II, Allen, L. E., Huard, T., \& Heyer, M. 2010, ApJ, 723, 1019

Heyer, M., \& Dame, T. M. 2015, ARA\&A, 53, 583

Jones, A. P., Köhler, M., Ysard, N., Bocchio, M., \& Verstraete, L. 2017, A\&A, 602, A46

Kainulainen, J., Beuther, H., Henning, T., \& Plume, R. 2009, A\&A, 508, L35

Khan, R. 2017, ApJS, 228, 5

Kirk, J. M., Gear, W. K., Fritz, J., et al. 2015, ApJ, 798, 58

Könyves, V., André, P., Men'shchikov, A., et al. 2010, A\&A, 518, L106

Lada, C. J., Alves, J., \& Lada, E. A. 1999, ApJ, 512, 250

Lada, C. J., Lada, E. A., Clemens, D. P., \& Bally, J. 1994, ApJ, 429, 694

Lada, C. J., Lombardi, M., \& Alves, J. F. 2009, ApJ, 703, 52

Lada, C. J., Lombardi, M., \& Alves, J. F. 2010, ApJ, 724, 687

Lada, C. J., Lombardi, M., Roman-Zuniga, C., Forbrich, J., \& Alves, J. F. 2013, ApJ, 778, 133

Lada, C. J., Margulis, M., Sofue, Y., Nakai, N., \& Handa, T. 1988, ApJ, 328,143

Larson, R. B. 1981, MNRAS, 194, 809

Leroy, A. K., Bolatto, A., Gordon, K., et al. 2011, ApJ, 737, 12

Licquia, T. C., \& Newman, J. A. 2015, ApJ, 806, 96

Lombardi, M., Alves, J., \& Lada, C. J. 2010, A\&A, 519, L7

Lombardi, M., Alves, J., \& Lada, C. J. 2015, A\&A, 576, L1

Lombardi, M., Bouy, H., Alves, J., \& Lada, C. J. 2014, A\&A, 566, A45

Madau, P., \& Dickinson, M. 2014, ARA\&A, 52, 415

McMullin, J. P., Waters, B., Schiebel, D., Young, W., \& Golap, K. 2007, in ASP Conf. Ser. 376, CASA Architecture and Applications, ed. R. A. Shaw, F. Hill, \& D. J. Bell (San Francisco, CA: ASP), 127

Menten, K. M., Reid, M. J., Forbrich, J., \& Brunthaler, A. 2007, A\&A, 474,515

Murray, N., \& Rahman, M. 2010, ApJ, 709, 424

Nieten, C., Neininger, N., Guélin, M., et al. 2006, A\&A, 453, 459

Nishimura, A., Tokuda, K., Kimura, K., et al. 2015, ApJS, 216, 18

Peng, Y.-j., Lilly, S. J., Kovač, K., et al. 2010, ApJ, 721, 193

Peretto, N., Lenfestey, C., Fuller, G. A., et al. 2016, A\&A, 590, A72

Planck Collaboration, Abergel, A., Ade, P. A. R., et al. 2014a, A\&A, 571, A11

Planck Collaboration, Ade, P. A. R., Aghanim, N., et al. 2014b, A\&A, 571, A9 
Riener, M., Faesi, C. M., Forbrich, J., \& Lada, C. J. 2018, A\&A, 612, A81

Sault, R. J., Teuben, P. J., \& Wright, M. C. H. 1995, in ASP Conf. Ser. 77, A Retrospective View of MIRIAD, ed. R. A. Shaw, H. E. Payne, \& J. J. T Hayes (San Francisco, CA: ASP), 433

Viaene, S., Baes, M., Tamm, A., et al. 2017, A\&A, 599, A64
Vogel, S. N., Boulanger, F., \& Ball, R. 1987, ApJL, 321, L145

Wakelam, V., Bron, E., Cazaux, S., et al. 2017, MolAs, 9, 1

Wang, S., Liu, J., Qiu, Y., et al. 2016, ApJS, 224, 40

Whitworth, A. P., Marsh, K. A., Cigan, P. J., et al. 2019, MNRAS, 489, 5436

Ysard, N., Köhler, M., Jones, A., et al. 2015, A\&A, 577, A110 